Article

\title{
Application of ATR-FT-MIR for Tracing the Geographical Origin of Honey Produced in the Maltese Islands
}

\author{
Jean Paul Formosa ${ }^{1}$, Frederick Lia ${ }^{1, * \mathbb{D}}$, David Mifsud ${ }^{2}$ and Claude Farrugia ${ }^{1} \mathbb{D}$ \\ 1 Department of Chemistry, University of Malta, 2080 MSD Msida, Malta; \\ jean.p.formosa.12@um.edu.mt (J.P.F.); claude.farrugia@um.edu.mt (C.F.) \\ 2 Department of Rural Sciences and Food Systems, University of Malta, 2080 MSD Msida, Malta; \\ david.a.mifsud@um.edu.mt \\ * Correspondence: fredericklia@gmail.com; Tel.: +356-99018310
}

Received: 24 April 2020; Accepted: 27 May 2020; Published: 1 June 2020

\begin{abstract}
Maltese honey has been produced, marketed, and sold as an exclusive local gourmet food product for countless years. Yet, thus far, no study has evaluated the individuality of this local food product. The evaluation of the parameters and properties which characterise the provenance and floral source of honey have been the subject of various studies worldwide, owing to the price and potential beneficial properties of this food product. Models analysing the potential of attenuated total reflection mid-infrared (ATR-FT-MIR) spectroscopy in discriminating and classifying local honey from that of foreign origin were investigated using 21 Maltese honey samples and 49 honey samples collected from abroad (Sicily, Greece, Sweden, Italy, France, Estonia and other samples of mixed geographical origin). Through a combination of spectroscopic techniques, spectral transformations, variable selection and partial least squares discriminant analysis (PLS-DA), chemometric models which successfully classified the provenance of local and non-local honey were developed. The results of these models were also corroborated with other classification and pattern recognition techniques, such as linear discriminate analysis (LDA), support vector machines (SVM) and feed-forward artificial neural networks (FF-ANN).
\end{abstract}

Keywords: Malta; honey; PLS-DA; LDA; SVM; FF-ANN; chemometrics; ATR-FT-MIR

\section{Introduction}

There is a considerable number of apiaries in Malta and Gozo, producing honey which is sought by locals and tourists alike, and valued for its unique taste and characteristics. At the moment, there is a sizable number of beekeepers selling Maltese honey directly or through local markets. However, the vast amount of honey being sold is raising suspicion that there might be cases of fraud, where the honey is either being mislabelled as Maltese honey, or else adulterated with sugar syrup and/or non-local honey.

The sugars fructose and glucose account for about $85 \%$ of honey solids, given that floral nectar is the source of honey sugars. Glucose and fructose are reported to be the only monosaccharides in honey, with an average concentration of $38 \% w / w$ for fructose and $31 \% w / w$ for glucose $[1,2]$. Oligosaccharides represent about $10 \%$ of the total honey weight [3] and are composed of several units, generally two to six units of glucose and fructose, with glycosidic linkages in different positions. Siddiqui [2] characterised 14 disaccharides and 11 trisaccharides, while Doner [4] showed that there is satisfactory evidence for the presence of 10-13 disaccharides and 8-9 trisaccharides. More recent studies have shown that 25 trisaccharides and 10 tetrasaccharides have been found in honey samples from Spain and New Zealand [5]. The composition of oligosaccharides in honey is related to the floral source, however, it is 
difficult to specify one sugar as a floral marker for honey. It has been suggested that the ratio of certain sugars, along with other parameters, can be used to differentiate between honeys [6].

There is a considerable number of apiaries in Malta and Gozo, producing honey which is sought by locals and tourists alike, and valued for its unique taste and characteristics. Malta has a long history

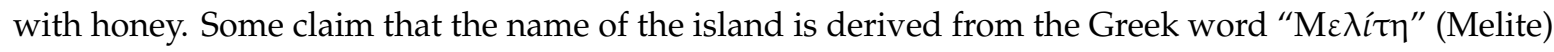
meaning 'honey-sweet', and furthermore, ancient bee hives dating to the time when the Romans occupied Malta (c. 200 BC) have been found. However, in recent times, there have been several concerns about the authenticity of Maltese honey being sold at local markets. At the moment, there are a sizable number of beekeepers selling Maltese honey directly or through local markets. However, the vast amount of honey being sold is raising suspicion that there might be cases of fraud, where the honey is either being mislabelled as Maltese honey or else adulterated with sugar syrup and/or non-local honey. Maltese honey is mostly collected by the Maltese honeybee Apis mellifera ruttneri, which is indigenous and endemic to the Maltese islands [7,8]. However, in recent years, this endemic species has become under threat, due to the importation of foreign queen bees from Sicily. While there is no published evidence on the effect of the mixing of the two honeybees, Apis mellifera ruttneri and Apis mellifera sicula, on the chemical and physical properties of Maltese honey, there are still significant threats to the local bee community and beekeepers. Firstly, there is the risk of the loss in biodiversity due to the wiping out of the indigenous honeybee, and furthermore, there is also a risk to the general public, due to the possible aggressive behaviour of the resulting hybrids.

Published literature on Maltese honey is lacking, and up till now, no in-depth chemical profiling has been performed on Maltese honey; in fact, the literature is mainly based on physicochemical parameters. These studies include HMF content, diastase and proline levels, and total phenolic content [9-11]. More recently, a comprehensive study with regards to a number of physicochemical parameters and sugar composition has also been published [11]. Furthermore, little or no comparison has been performed with regards to the physicochemical and chemical properties of Maltese honey with honey samples from other regions of the world.

Infrared (IR) spectroscopy is a technique used frequently in food analysis for authentication, quantification and detection of adulteration [12], and has been favoured as a rapid, non-destructive, cheap and reagent free technique in the food industry $[13,14]$. In combination with chemometrics, IR spectroscopy was successfully applied for the determination of different attributes and adulterants [15] in several food samples, including juice analysis [16]; alcoholic beverages [7,17-19] must and wine analysis [9,20,21], polymethoxylated flavone of orange oil residues [22], organic acids and carbohydrates determination in fruits [23], and characterisation of olive oil and olive pulp [24,25].

Near-infrared (NIR) and mid-infrared (MIR) methods, coupled with signal processing and chemometric techniques, have been extensively developed in recent years, for quality control and the authentication of honey samples [26], including numerous attenuated total reflection mid-infrared (ATR-FT-MIR) methods. Several methods have been developed for the detection of sugar syrups in honey, particularly using partial least squares discriminant analysis (PLS-DA) [20,27-33], and through the use of principle component analysis (PCA), linear discriminate analysis (LDA) and artificial neural networks (ANN) [34]. PLS regression and principal component regression (PCR) have also been use in conjunction with ATR-FT-MIR spectroscopy for the quantification of sugars in honey; namely, glucose, fructose, sucrose, and maltose, melezitose and turanose [35-39].

ATR-FT-MIR methods have also been successfully employed in conjunction with chemometric methods, including PCA and LDA, for differentiation of botanical origin [36-42]. Hennessy et al. [40], used several signal processing methods on MIR spectra of Corsican and non-Corsican honey, in conjunction with FDA (factorial discriminant analysis) and PLS analysis for classification. NIR spectroscopy and signal processing methods were also shown to be essential tools in conjunction with SIMCA (soft independent modelling of class analogy) and PLS for the geographical classification of Irish, Mexican, and Spanish, Argentinean, Czech, Hungarian, and Irish honey samples [41,42]. These studies all employ the use of spectral transformations prior to multivariate analysis. Spectral transformations are especially important 
when applied to IR data, in order to remove spectral artefacts such as baseline shifts and multicollinearity and can also reveal 'hidden' information by emphasising small spectral variations. The aim of this research is the analysis of Maltese and foreign honey by ATR-FT-MIR, alongside several data treatment and pattern recognition techniques. The study also aims to identify which spectral transformation or combination of them are more adequate for their discrimination.

\section{Materials and Methods}

\subsection{Honey Samples}

A total of 70 samples were collected and 21 local samples were directly collected from Maltese and Gozitan beekeepers post honey harvest, between the period of 2015 and 2016. Further details are presented in the Supplementary Materials Figure S1 and Table S1. Overall, 49 foreign samples were collected from different Mediterranean countries directly from various international beekeepers associations. Furthermore, foreign samples sold from local supermarkets were also included. All samples were kept in the dark at $20^{\circ} \mathrm{C}$ until analysis.

\subsection{FTIR Method}

Prior to scanning, honey samples were homogenized after heating to $30^{\circ} \mathrm{C}$ for one hour, followed by stirring. A Shimadzu IR-Affinity 1 equipped with a Silver Gate Zn/Se ATR was used for spectral acquisition. The instrument was set to acquire 32 scans per spectrum at a resolution of $4 \mathrm{~cm}^{-1}$ in the range of $4000-550 \mathrm{~cm}^{-1}$. In order to obtain a spectrum with a high signal to noise ratio and to reduce the error in the baseline, the instrument was blanked before each sample and each spectrum was run in triplicate. The mean of these replicates was then used in the following data analysis procedures.

\subsection{Chemometric Analysis}

Initial data treatment included first removing the region between 2800 and $1800 \mathrm{~cm}^{-1}$ in which no peaks arise. The honey sample matrix contains a negligible amount of chemicals which have active bands in this region [29]. The spectra were also trimmed at the ends to a range of $740-3600 \mathrm{~cm}^{-1}$, in order to remove regions which contained a significant amount of noise and no relevant chemical data (Figure 1). The spectra obtained were subjected to different spectroscopic signal processing techniques which were evaluated and compared. These include subtraction of a linear baseline, multiplicative scatter correction (MSC), orthogonal signal correction (OSC), standard normal variate (SNV), and first and second derivative Savitzky-Golay transformations. The effect of the different spectral transformations on the final classification outcomes was compared to those obtained without any signal processing.

Several spectral transformations were applied prior to statistical analysis using the Unscrambler $X$ (CAMO A/S, Oslo, Norway). Smoothing was the first transformation applied to the IR spectra. There are a variety of smoothing algorithms which can be applied to spectra, including moving average, Gaussian, median and Savitzky-Golay. The spectrum with maximum smoothness and minimum distortion from the original signal was selected, thus, a compromise between noise reduction and retention of information was evaluated. This was also further confirmed by PLS-DA analysis, which showed that the best improvement and highest explained variance out of all smoothed spectra was obtained when using a median filter with a gap size of three.

MSC, OSC, detrending, deresolving, SNV, along with a combination of SNV and detrending filters were then applied to the smoothed spectral data, in order to determine their effect on the misclassification rate and the RMSE error. Furthermore, first and second Savitzky-Golay derivative transformations were applied to the spectra in the region with a gap size of 7 points and a polynomial order of two. 


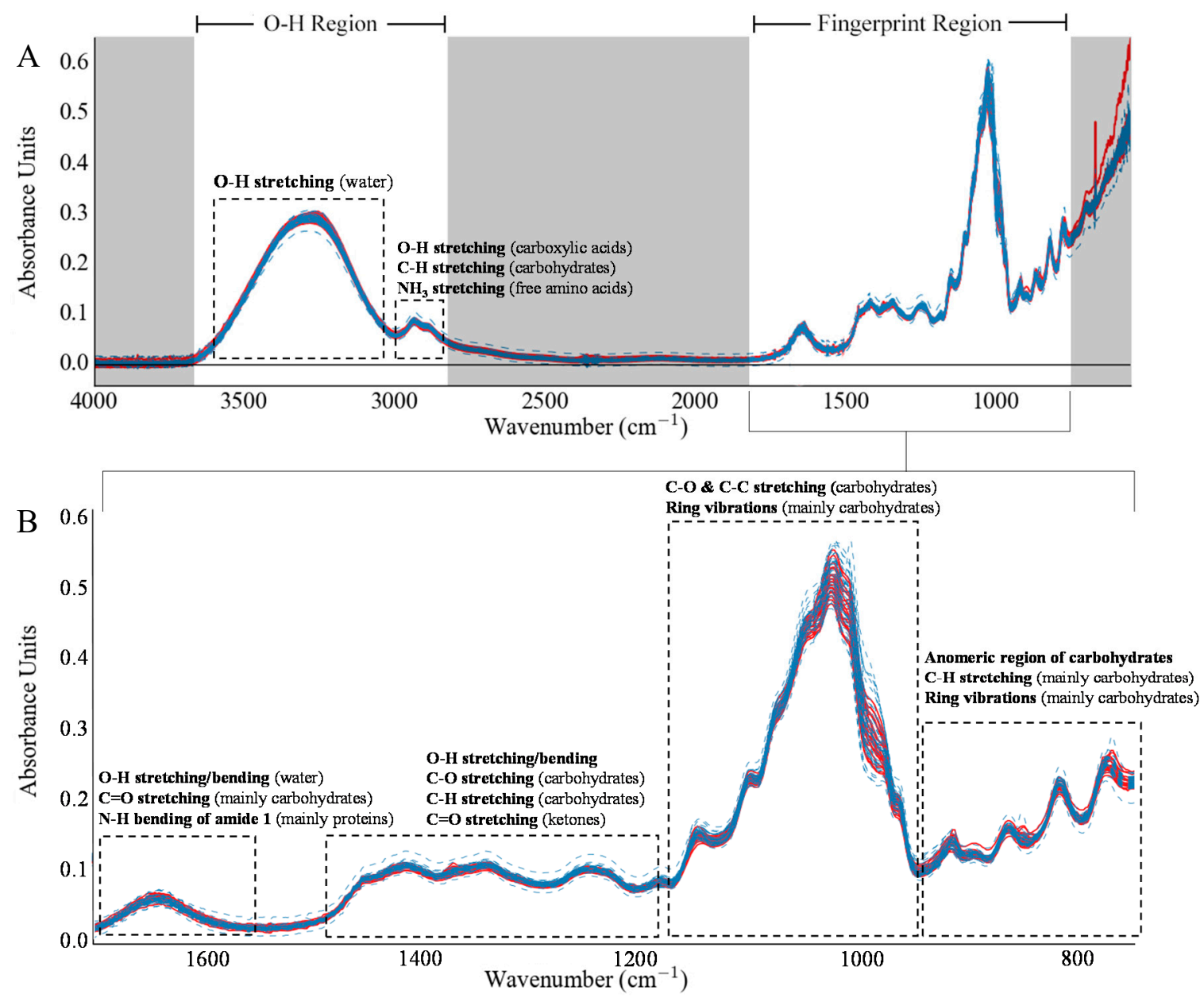

Figure 1. (A) IR Spectra of all tested samples (continuous red lines represent local samples and dashed blue lines represent foreign samples); regions which were not used in this study are shaded in grey. (B) Expansion of the fingerprint region highlighting the major peaks identified in this study.

Principal component analysis (PCA) was carried out on the data, in order to hint at possible outliers or any possible clustering of the Maltese samples present within the data set. The supervised chemometric treatment was performed using PLS-DA, in order to classify the geographical origin with regards to Maltese and non-Maltese samples. The former samples were assigned a dummy variable of 1 , while the latter were assigned a value of 0 . Samples with a predicted value of $>0.5$ were thus labelled as foreign, while the remaining samples were labelled as local. PLS-DA analysis was carried out on the whole data set, using leave one out cross-validation (LOOCV), after which PLS-DA was repeated using excluded rows validation (ERV), with the exclusion of one third of the samples from each class to assess for model overfitting. The RMSE for the model was calculated as shown below, in order to further assess the accuracy of the model. Where $y_{\text {pred }}$ corresponds the value between 0 and 1 generated by the model, whilst $y_{\text {ref }}$ corresponds to the dummy variable to which the honey was assigned.

$$
\text { RMSE }=\sqrt{\frac{\sum_{i=1}^{n}\left(y_{\text {pred }}-y_{\text {ref }}\right)^{2}}{n}}
$$

The optimum model for each transformation was chosen after an assessment of the PLS-DA model parameters. The classification accuracy of the LOOCV and ERV models, explained as X and Y variance, RMSE and number of factors were used to evaluate the performance of the chemometric models. 


\subsubsection{Variable Selection}

Once the optimum number of factors is determined, the data points which had a VIP (variable importance in projection) $>0.8$ were then used to develop subsequent PLS-DA models. The VIP score is a measure of a variable's importance in the PLS model. It represents the contribution of a variable to the PLS model and is determined through a weighted sum of the squared correlations between the model components and the original variable. A value of less than 0.8 is typically considered to be a small VIP, and thus, a candidate for deletion from the model [43]. VIP scores are useful in understanding X space predictor variables that best explain y variance. VIP scores give an estimate of the contribution of a given predictor to a PLS regression model [44].

Furthermore, stepwise linear canonical discriminant analysis (SLC-DA), as implemented within JMP, was also used, in order to reduce the number of variables used in PLS-DA models. A stepwise analysis allows for the manual selection of variables used to build the linear model up to a maximum number of entries $(n-1)$, where $\mathrm{n}$ is the number of samples in the sample set. The model containing the most discriminant variables was selected on the basis of a low F-ratio and a high $p$-Value.

\subsubsection{Statistical Analysis}

Feed-forward artificial neural networks (FF-ANN), support vector machines (SVM) and linear discriminant analysis (LDA) were implemented as a further corroboration and validation to the PLS-DA models. FF-ANN, LDA and SVM analysis were carried using a Python script and the 'scikit-learn' Machine Learning toolbox for Python [45]. FF-ANN models were implemented on data without variable selection, whilst SVM and LDA classification methods were applied on data with SLC-DA model selection.

SVM models have no limit on the number of variables which can be used in a model. Nonetheless, SVM models require a computationally intensive grid search and thus analysis were performed on SLC-DA selected variables. Models for LDA were also performed on the SLC-DA selected variable, as this classification technique is usually limited to small number of variables, which must be less than the number of samples in each class. On the other hand, FF-ANN models are suited for modelling data with a large number of variables, and thus were used to model data with no variable selection. In all cases the models were validated both using ERV in a similar fashion to PLS-DA models.

The aforementioned statistical analysis and variable selection steps were also carried out on the fingerprint region (760-1400 $\mathrm{cm}^{-1}$ ), in order to determine the effect of using this portion of the spectrum only on the prediction rate and RMSE of the models.

\section{Results and Discussion}

\subsection{Geographical Classification Using ATR-FT-MIR}

Monosaccharides, water, and other sugars are the main components in honey, thus, most of the spectral peaks observed in honey IR spectra appertain to vibrational modes exhibited from sugars and water [23]. Water in honey shows up as a very distinct broad peak between $3500-3000 \mathrm{~cm}^{-1}$ in the honey IR spectrum (Figure 1). Additionally, a peak is observed between $3000-2800 \mathrm{~cm}^{-1}$, which arises from vibrational modes of carbohydrates [32], carboxylic acids [46] and amino acids [29]. The region between $1700-1600 \mathrm{~cm}^{-1}$ shows the vibrational modes from water [47], carbohydrates [32] and the amide I band [48]. The peaks within the fingerprint region $\left(1500-700 \mathrm{~cm}^{-1}\right)$ are attributed to various vibrational modes of carbohydrates and ketones. The vibrations that occur between 1200 and $1300 \mathrm{~cm}^{-1}$ are attributed to the presence of $-\mathrm{C}-\mathrm{O}$ bonds, whilst that at $1750 \mathrm{~cm}^{-1}$ accounts for the carboxylic acid functionalities $(\mathrm{C}=\mathrm{O})$ of various carbohydrates. The bands observed in the range between 1150 and $995 \mathrm{~cm}^{-1}$ are attributed to the stretching and bending vibrations of $\mathrm{C}-\mathrm{O}, \mathrm{C}-\mathrm{H}$ and $\mathrm{C}-\mathrm{OH}$ vibrations arising from carbohydrates. [34,36].

The first data handling stage involved the removal of the region between $2700 \mathrm{~cm}^{-1}$ and $1800 \mathrm{~cm}^{-1}$, as it contained no IR bands which are expected to show up from the honey sample matrix; for simplicity's 
sake, this region will be referred to as the 'whole spectrum' henceforth. The median filter transformation was particularly effective in removing any noise generated by the ATR-FTIR while leaving any slight spectral variations intact. Further spectral transformations were then applied to median filter smoothed MIR spectra, since the application of some spectral transformations such as derivative transformations tended to accentuate any noise present.

A visual inspection of the MIR spectra (Figure 1) and the resulting spectral transformations revealed no regions which offer discrimination between Maltese and non-Maltese samples. Furthermore, PCA identified no outliers within the dataset for the untreated spectra, or when the spectra were subjected to spectral transformations. Through PCA, no samples were observed to cluster according to geographical origin. This result is not unexpected, since the honey samples being tested do not differ only by geographical origin, since other sources of variability, such as botanical origin, were present. PCA analysis is presented in Supplementary Materials Figures S2 and S3.

\subsubsection{PLS-DA and Variable Selection}

PLS-DA was used as the primary statistical model for sample classification and the prediction of Maltese and non-Maltese samples (Table 1). In the majority of the cross validated PLS models on the 'whole' MIR spectra (Table 1), no samples were misclassified, except for the second derivative transformation model, which exhibited an accuracy of 98.6\%. The RMSEs for all the LOOCV models were considerably low, wherein the RMSE will effectively describe the average distances of the predicted sample towards the dummy classification system used.

Table 1. Results from partial least squares discriminant analysis (PLS-DA) models applied to spectral transformations of attenuated total reflection mid-infrared (ATR-FT-MIR) spectra and variable selection procedures. $(\mathrm{MF}=$ Median Filter, 1 st $=$ Savitzky-Golay first derivative, 2 nd $=$ Savitzky-Golay second derivative, $\mathrm{DR}=$ De-resolve, $\mathrm{DT}=$ De-trend, $\mathrm{MSC}=$ Multiplicative Scatter Correction, $\mathrm{OSC}=$ Orthogonal Signal Correction, $\mathrm{QN}=$ Quantile Normalise, SNV = Standard Normal Variate, SNVDT = combination of SNV and DT transformations \#F = number of latent factors extracted from the PLS model).

\begin{tabular}{|c|c|c|c|c|c|c|c|c|c|c|c|}
\hline \multirow{3}{*}{$\begin{array}{l}\text { Variable } \\
\text { Selection }\end{array}$} & \multirow{3}{*}{ Pre-Treatment } & \multicolumn{5}{|c|}{ Whole Spectrum } & \multicolumn{5}{|c|}{ Fingerprint Region } \\
\hline & & \multirow{2}{*}{$\# \mathbf{F}$} & \multicolumn{2}{|c|}{ LOOCV } & \multicolumn{2}{|c|}{ ERV } & \multirow{2}{*}{$\# \mathrm{~F}$} & \multicolumn{2}{|c|}{ LOOCV } & \multicolumn{2}{|c|}{ ERV } \\
\hline & & & Accuracy \% & RMSE & Accuracy \% & RMSE & & Accuracy \% & RMSE & Accuracy $\%$ & RMSE \\
\hline \multirow{7}{*}{ None } & 1st DSG & 5 & 100.0 & 0.120 & 92.8 & 0.248 & 8 & 100.0 & 0.100 & 97.1 & 0.192 \\
\hline & 2nd DSG & 4 & 98.6 & 0.166 & 95.7 & 0.261 & 6 & 100.0 & 0.184 & 97.1 & 0.257 \\
\hline & DR & 14 & 100.0 & 0.170 & 97.1 & 0.205 & 10 & 97.1 & 0.214 & 100.0 & 0.218 \\
\hline & OSC & 13 & 100.0 & 0.110 & 95.7 & 0.197 & 9 & 100.0 & 0.186 & 100.0 & 0.193 \\
\hline & $\mathrm{QN}$ & 8 & 100.0 & 0.135 & 94.2 & 0.222 & 9 & 100.0 & 0.111 & 97.1 & 0.186 \\
\hline & SNV & 10 & 100.0 & 0.101 & 98.6 & 0.210 & 10 & 100.0 & 0.126 & 100.0 & 0.169 \\
\hline & SNVDT & 9 & 100.0 & 0.160 & 97.2 & 0.219 & 10 & 100.0 & 0.114 & 100.0 & 0.167 \\
\hline \multirow{6}{*}{ VIP } & MF & 15 & 100.0 & 0.047 & 100.0 & 0.111 & 12 & 100.0 & 0.106 & 97.1 & 0.171 \\
\hline & 1st DSG & 6 & 100.0 & 0.089 & 97.1 & 0.168 & 10 & 100.0 & 0.069 & 97.1 & 0.181 \\
\hline & OSC & 13 & 100.0 & 0.104 & 95.7 & 0.178 & 12 & 100.0 & 0.111 & 95.7 & 0.174 \\
\hline & $\mathrm{QN}$ & 13 & 100.0 & 0.025 & 95.7 & 0.187 & 10 & 100.0 & 0.091 & 97.1 & 0.180 \\
\hline & SNV & 10 & 100.0 & 0.176 & 98.6 & 0.183 & 10 & 100.0 & 0.125 & 100.0 & 0.164 \\
\hline & SNVDT & 12 & 100.0 & 0.073 & 94.2 & 0.227 & 10 & 100.0 & 0.111 & 100.0 & 0.163 \\
\hline \multirow{9}{*}{ SLCDA } & MF & 15 & 100.0 & 0.089 & 100.0 & 0.133 & 15 & 100.0 & 0.094 & 100.0 & 0.149 \\
\hline & 1st DSG & 15 & 100.0 & 0.047 & 100.0 & 0.152 & 15 & 100.0 & 0.077 & 97.1 & 0.201 \\
\hline & 2nd DSG & 14 & 100.0 & 0.048 & 97.1 & 0.163 & 15 & 100.0 & 0.100 & 98.6 & 0.155 \\
\hline & DR & 15 & 100.0 & 0.136 & 94.2 & 0.301 & 15 & 100.0 & 0.138 & 100.0 & 0.188 \\
\hline & DT & 15 & 100.0 & 0.041 & 98.6 & 0.122 & 15 & 100.0 & 0.064 & 100.0 & 0.148 \\
\hline & MSC & 15 & 100.0 & 0.089 & 100.0 & 0.128 & 15 & 100.0 & 0.076 & 100.0 & 0.139 \\
\hline & OSC & 15 & 100.0 & 0.102 & 100.0 & 0.144 & 15 & 100.0 & 0.109 & 98.6 & 0.141 \\
\hline & QN & 15 & 100.0 & 0.042 & 100.0 & 0.087 & 15 & 100.0 & 0.077 & 100.0 & 0.085 \\
\hline & SNV & 15 & 100.0 & 0.047 & 100.0 & 0.111 & 10 & 100.0 & 0.126 & 100.0 & 0.169 \\
\hline
\end{tabular}


ERV PLS-DA models exhibited a decrease in the \% accuracy, accompanied with an increase in the RMSE; this is expected, since the models relied on smaller number of samples. Ideally, well-calibrated models should exhibit little or no change on moving from cross-validated models to excluded rows validated models, whereas a significant drop in accuracy and an increase in the RMSE often suggests that the model is over-fitted. An over-fitted model will not solely describe the systematic variation in a model, but will also describe some of the random variation within the dataset and will give inaccurate predictions. For most of the models (Table 1), the drop in prediction accuracy was not very large, since most models show an accuracy $>95 \%$, which suggests that these models were well-calibrated.

The use of VIP scores for data reduction in PLS shows a notable improvement with regards to the classification rate in both the cross validated and excluded rows models. Furthermore, a markedly larger improvement was also observed when using SLC-DA for variable selection (Table 1), where all the internally validated PLS-DA models exhibited no misclassifications and considerably lower RMSEs than the PLS models without variable selection and VIP variable selection. An example of such a plot of VIP and SLCDA scores for the media transformation is included in Figure 2b,c.

The externally validated PLS-DA models also showed a significant improvement when SLCDA is used, with most models showing no misclassifications. The PLS-DA model improvement can be attributed to the large amount of variable reduction, from around 550 variables to around $20-40$ variables, wherein the amount of redundant and collinear variables is reduced (Figure 2).

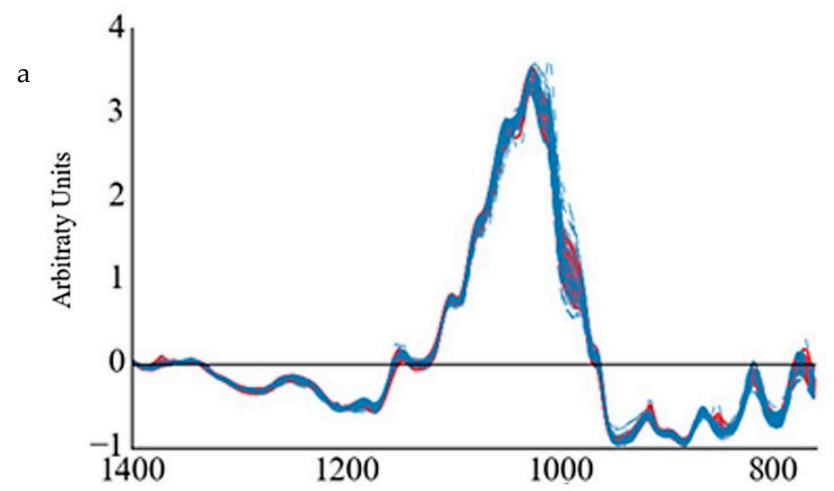

b
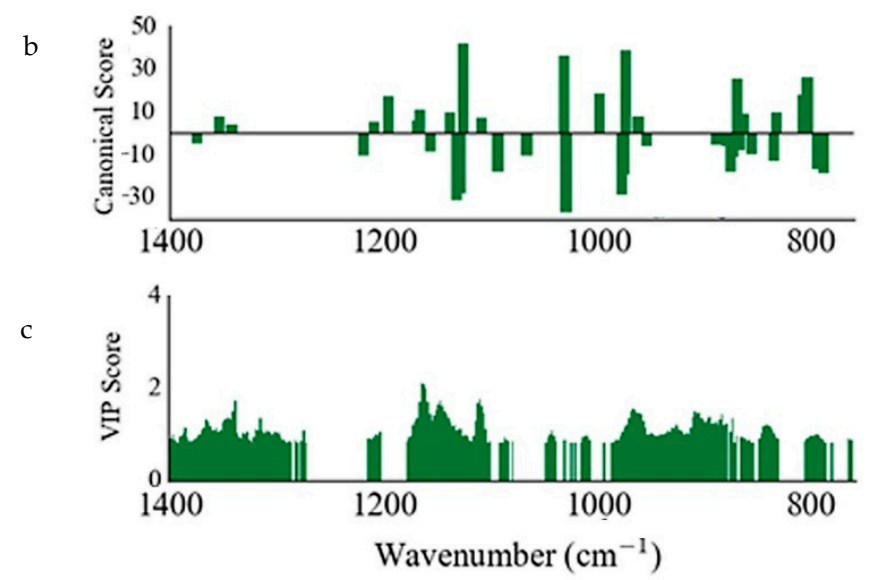

Figure 2. (a) SNVDT transformed ATR-FT-MIR spectra of fingerprint region of all honey samples (continuous red lines represent local samples and dashed blue lines represent foreign samples), (b) stepwise linear canonical discriminant analysis (SLC-DA) canonical scores and (c) VIP scores $(>0.8)$ obtained from the variable selection procedures performed on the transformed spectra in (a).

Generally, there is a marked improvement when only the fingerprint region was used to develop PLS-DA model (Table 1), when compared to models on the 'whole' spectra. A similar trend towards a drop in the prediction accuracy for the excluded rows validation is also observed in this case. The OSC, 
SNV and a combination of SNV and detrending transformations generated PLS-DA models, which correctly classify all the samples through external validated models, without any variable selection as show in Figure 3. This highlighted the effectivity of these transformations when used in conjunction with PLS-DA for the geographical profiling of Maltese and non-Maltese samples.

The removal of the variables which had VIP scores less than 0.8 on the fingerprint region generally decreased the RMSE in the internally validated PLS-DA models, which maintained their classification accuracy. The PLS-DA models using external validation were also shown to generally improve, whereas the models using OSC and median filter transformations showed an increase in the number of misclassifications. Moreover, PLS-DA models using the variables selected by SLC-DA (Table 1) generally showed a marked improvement on the models using no variable selection and VIP variable selection, except for the model using a de-resolve transformation.

Furthermore, apart from a higher accuracy and lower RMSE, PLS-DA models on spectral transformations of the fingerprint region generally exhibited a higher \% explained variance for the predictor matrices when compared to the 'whole' spectra. This was generally true in the case of PLS-DA models without variable selection and models which used VIP scores for variable selection. Nonetheless, the performance of the PLS models using the SLC-DA variables was similar in both cases (Table 1). The lack of improvement over using the fingerprint region versus the whole spectrum for analysis in the case of SLC-DA, is due to the fact that SLC-DA is very effective at variable selection, thus removing any redundant variables present in the region from $3600-2800 \mathrm{~cm}^{-1}$.

In light of these findings, it can be concluded that the fingerprint region is more suited for the differentiation of local and foreign samples using PLS-DA analysis, since it carries more relevant information and still gives very good classification accuracy without the need of variable selection. Lastly, while the model parameters give a good indication of the performance of the PLS-DA models, at this stage, they should not be used to single out the best performing transformation method for classifying Maltese and non-Maltese honey using PLS-DA. This is because the samples only represent a small set of local honey and an even smaller set of non-local honey, and thus different samples might be better represented using different transformations. Nevertheless, the high classification rates and low error values highlight the potential application of ATR-FT-MIR spectroscopy and spectral transformations in combination with PLS-DA for the routine classification of local and foreign honey.

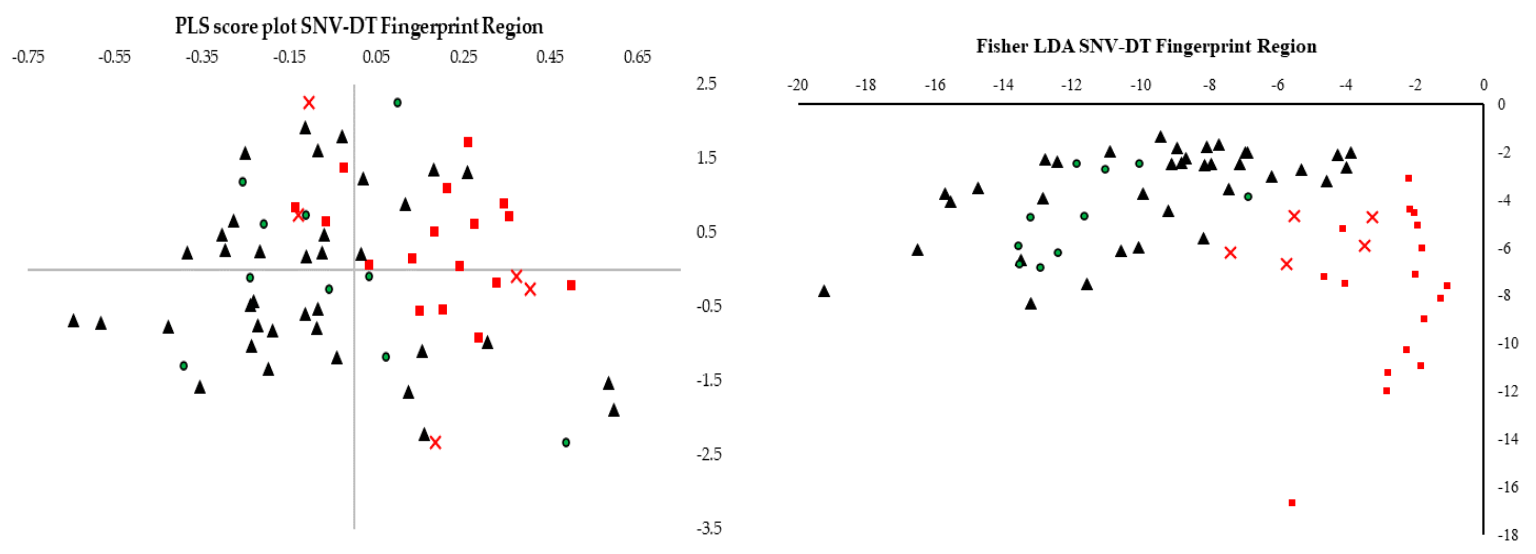

(a)

(b)

Figure 3. (a) the PLS score plot obtained using SNVDT transformed ATR-FT-MIR complete spectra under ERV. (b) Linear discriminate analysis performed on the extracted PLS scores. (ム) Foreign honey samples used in the training set (घ); Maltese honey samples used in the training set $(\bullet)$; foreign honey samples used in the validation set (x); Maltese honey samples used in the validation set. Maltese samples are depicted in red, whilst the foreign samples are depicted in black. 


\subsubsection{Other Models}

Excluded row FF-ANN models (Table 2) on spectral transformations of the whole MIR spectra showed a slight improvement in the classification rate when compared to their respective PLS-DA models. Conversely, the excluded rows validated FF-ANN models performed on spectral transformations of the fingerprint region showed an increase in the number of misclassifications for nearly all the spectral transformations, except for models derived from MSC and SNV transformed spectra. This infers that FF-ANN models were more effective at extracting information from the 'whole' spectra for classification than from the fingerprint region. A similar trend was observed in the excluded rows SVM model results (Table 3), where the classification rate was generally higher for SVM models of spectral transformation on the 'whole' spectra than for spectral transformation in the fingerprint region; thus, the same inference can be made.

Table 2. Summary of FF-ANN Model performance with no variable selection on the 'whole' spectrum and on fingerprint region only.

\begin{tabular}{ccccc}
\hline \multirow{2}{*}{$\begin{array}{c}\text { Data Pre-Treatment } \\
\text { Method }\end{array}$} & \multicolumn{2}{c}{ Whole } & \multicolumn{2}{c}{ Fingerprint } \\
\cline { 2 - 5 } & Accuracy (\%) & RMSE & Accuracy (\%) & RMSE \\
\hline Median Filter & 97.1 & 0.1299 & 97.1 & 0.1761 \\
First Derivative (SG) & 92.8 & 0.2429 & 95.7 & 0.1717 \\
Second Derivative (SG) & 95.7 & 0.2186 & 95.7 & 0.1915 \\
Deresolve & 100.0 & 0.0609 & 98.6 & 0.0791 \\
Detrending & 95.6 & 0.1648 & 97.1 & 0.1713 \\
MSC & 98.6 & 0.1019 & 98.6 & 0.1198 \\
OSC & 97.1 & 0.1604 & 97.1 & 0.1636 \\
Quantile Normalise & 95.7 & 0.1940 & 95.7 & 0.1958 \\
SNV & 98.6 & 0.1142 & 98.6 & 0.1175 \\
SNVDT & 98.6 & 0.0841 & 97.1 & 0.1427 \\
\hline
\end{tabular}

Nevertheless, the high classification rates obtained by most excluded rows validated FF-ANN, SVM and PLS-DA models, further corroborating the use of MIR spectra and spectral transformations as a method for the classification of local and foreign samples. The PLS-DA model results were also further corroborated by the LDA model results (Table 3), which showed no misclassifications in most instances. In fact, LDA models commonly showed better classification rates than the corresponding PLS-DA models. These results highlight the potential use of open source pattern recognition packages for further development and implementation in chemometric applications.

Table 3. Summary of SVM and LDA Model performance with no variable selection on the 'whole' spectrum and on fingerprint region only.

\begin{tabular}{ccccccc}
\hline \multirow{2}{*}{$\begin{array}{c}\text { Data Pre-Treatment } \\
\text { Method }\end{array}$} & \multicolumn{3}{c}{ LDA } & & SVM & \multicolumn{3}{c}{ FDA } & & SVM \\
\cline { 2 - 7 } & Accuracy (\%) & RMSE & Accuracy (\%) & Accuracy (\%) & RMSE & Accuracy (\%) \\
\cline { 2 - 7 } & 100.0 & 0.0003 & 100.0 & 98.6 & 0.0842 & 98.6 \\
Median Filter & 100.0 & 0.0000 & 100.0 & 100.0 & 0.0128 & 97.1 \\
First Derivative (SG) & 100.0 & 0.0000 & 100.0 & 98.6 & 0.1095 & 92.8 \\
Second Derivative & 98.6 & 0.1205 & 95.7 & 100.0 & 0.0658 & 98.6 \\
(SG) & 100.0 & 0.0000 & 100.0 & 100.0 & 0.0000 & 98.6 \\
Deresolve & 100.0 & 0.0003 & 100.0 & 100.0 & 0.0000 & 98.6 \\
Detrending & 100.0 & 0.0125 & 100.0 & 100.0 & 0.0003 & 100.0 \\
MSC & 100.0 & 0.0000 & 100.0 & 100.0 & 0.0000 & 98.6 \\
OSC & 100.0 & 0.0000 & 100.0 & 100.0 & 0.0000 & 100.0 \\
Quantile Normalise & 100.0 & 0.0000 & 100.0 & 100.0 & 0.0000 & 98.6 \\
SNV & & & & & &
\end{tabular}




\section{Conclusions}

Most spectral transformations on ATR-FT-MIR data in combination with PLS-DA were shown to be very effective in classifying local and non-local honey samples. Furthermore, the use of the fingerprint region for classifying samples was shown to be more effective in PLS-DA models using no variable selection and VIP variable selection. The use of SLC-DA for variable selection was also shown to be significantly effective in decreasing the number of misclassifications, both when using the 'whole' spectrum and when using the fingerprint region.

FF-ANN, SVM and LDA models were shown to offer similar classification rates to PLS-DA models and this thus corroborates the results obtained from the PLS-DA models and places confidence in the use of ATR-FT-MIR methods in conjunction with spectral transformations, for the classification of Maltese and foreign honey samples. These results highlight the potential of these methods to be further developed, for the detection of adulteration and for more in-depth profiling and classification of Maltese honey. Furthermore, the results obtained highlight the effectiveness of chemometric and pattern recognition-based approaches, in order to quickly and reliably test the authenticity of honey samples. These promising results should thus serve as an incentive for more research to be done on developing a more extensive model, using other techniques such as fluorescence spectroscopy and NMR.

Supplementary Materials: The following are available online at http://www.mdpi.com/2304-8158/9/6/710/s1, Figure S1: Map of Maltese islands highlight locality of honey samples in Table S1, Figure S2: Two component plot from PCA on Median filtered data in the region between 760 and $1400 \mathrm{~cm}^{-1}$, Figure S3: Loading plot for the first two component of PCA on Median filtered data in the region between 760 and $1400 \mathrm{~cm}^{-1}$, Table S1: Sample code, locality and date of harvest for local samples.

Author Contributions: J.P.F., data acquisition, research paper conceptualization, methodology, software, validation, formal analysis, investigation, data curation, writing-original draft preparation, writing-review and editing, and funding acquisition; F.L., data acquisition, methodology, software, validation, writing-review and editing, conceptualization, writing — original draft preparation, D.M., writing_review and editing, and supervision; C.F., conceptualization, software, writing - original draft preparation, formal analysis supervision and project administration. All authors have read and agreed to the published version of the manuscript.

Funding: This work was supported by the ENDEAVOR Scholarships scheme (ESF.01.015). The scholarship is part-financed by the European Union-European Social Fund.

Acknowledgments: Greek samples were provided by Maria Bouga (Agricultural University of Athens). Swedish, French, and Estonian samples were obtained during a beekeeping conference in Malmo, Sweden in March 2016, with the collaboration of Walter Haefeker (President of the European Professional Beekeepers association), Thomas Dahl and Lotta Fabricius Kristiansen (Swedish Beekeepers Association). The Estonian samples were provided by Tago Holsting (Estonian Beekeepers Association).

Conflicts of Interest: The authors declare no conflict of interest.

\section{References}

1. Codex Alimentarius, Draft Revised Standard for Honey; Alinorm 01/25 19-26; Food and Agriculture Organization of the United Nations: Rome, Italy, 2001.

2. Siddiqui, I.R. The Sugars of Honey. Adv. Carbohydr. Chem. Biochem. 1971, 25, 285-309.

3. Sanz, M.L.; Polemis, N.; Morales, V.; Corzo, N.; Drakoularakou, A.; Gibson, G.R. In vitro investigation into the potential prebiotic activity of honey oligosaccharides. J. Agric. Food Chem. 2005, 53, 2914-2921. [CrossRef] [PubMed]

4. Doner, L.W. The sugars of honey-a review. J. Sci. Food Agric. 1977, 28, 443-456. [CrossRef] [PubMed]

5. Ruiz-Matute, A.I.; Rodríguez-Sánchez, S.; Sanz, M.L.; Martínez-Castro, I. Detection of Adulterations of Honey with High Fructose Syrups from Inulin by GC Analysis. J. Food Compos. Anal. 2010, 23, 273-276. [CrossRef]

6. Kaškonienè, V.; Venskutonis, P.R.; Čeksterytė, V. Carbohydrate composition and electrical conductivity of different origin honeys from Lithuania. Food Sci. Technol. 2010, 43, 801-807. [CrossRef]

7. Sheppard, W.S.; Arias, M.C.; Grech, A.; Meixner, M.D. Apis mellifera ruttneri, a new honey bee subspecies from Malta. Apidologie 1997, 28, 287-293. [CrossRef] 
8. Zammit-Mangion, M.; Meixner, M.; Mifsud, D.; Sammut, S.; Camilleri, L. Thorough morphological and genetic evidence confirm the existence of the endemic honey bee of the Maltese Islands Apis mellifera ruttneri: Recommendations for conservation. J. Apic. Res. 2017, 56, 514-522. [CrossRef]

9. Attard, E.; Mizzi, J. Physicochemical characterization of Gozitan Honey. IJFS 2013, 2, 180-187. [CrossRef]

10. Attard, E.; Bugeja Douglas, A.; Camilleri, C. Molecular characterization of Maltese honey: Diastase and proline levels changes in Maltese honey seasons. Farm Anim. Proteom. 2013, 2013, 266-269.

11. Attard, E.; Bugeja Douglas, A. Physicochemical characterization of Maltese honey. In Honey Analysis; InTech: Rijeka, Croatia, 2017.

12. Rodriguez-Saona, L.E.; Allendorf, M.E. Use of FTIR for rapid authentication and detection of adulteration of food. Annu. Rev. Food Sci. Technol. 2011, 2, 467-483. [CrossRef]

13. Valand, R.; Tanna, S.; Lawson, G.; Bengtström, L. A review of Fourier Transform Infrared (FTIR) spectroscopy used in food adulteration and authenticity investigations. Food. Addit. Contam. Part A 2020, 37, 19-38. [CrossRef] [PubMed]

14. Chalmers, J.M.; Griffiths, P.R. Handbook of Vibrational Spectroscopy; John Wiley and Sons: Chichester, UK, 2002; Volume 5.

15. Li-Chan, E.; Chalmers, J.M.; Griffiths, P. Applications of Vibrational Spectroscopy in Food Science: Instrumentation and Fundamental Applications; John Wiley and Sons: Chichester, UK, 2010.

16. Jha, S.N.; Gunasekaran, S. Authentications of sweetness of mango juice using Fourier transform infraredattenuated total reflection spectroscopy. J. Food Eng. 2010, 101, 337-342. [CrossRef]

17. Cocciardi, R.A.; Ismail, A.A.; Sedman, J. Investigation of the potential utility of single-bounce attenuated total reflectance Fourier transform infrared spectroscopy in the analysis of distilled liquors and wines. J. Agric. Food Chem. 2005, 53, 2803-2809. [CrossRef] [PubMed]

18. Lachenmeier, D.W. Rapid quality control of spirit drinks and beer using multivariate data analysis of Fourier transform infrared spectra. Food Chem. 2007, 101, 825-832. [CrossRef]

19. Moreira, J.L.; Marcos, A.M.; Barros, P. Analysis of Portuguese wine by Fourier transform infrared spectrometry. J. Viticul. Enol. 2002, 17, 27-33.

20. Fernandez, K.; Agosin, E. Quantitative analysis of red wine tannins using Fourier-transform mid-infrared spectroscopy. J. Agric. Food Chem. 2007, 55, 7294-7300. [CrossRef]

21. Villiers, A.; Alberts, P.; Tredoux, A.G.; Nieuwoudt, H.H. Analytical techniques for wine analysis: An African perspective; a review. Anal. Chim. Acta 2012, 730, 2-23. [CrossRef]

22. Manthey, J.A. Fourier transform infrared spectroscopy analysis of the polymethoxylated flavones content of orange oil residues. J. Agric. Food Chem. 2006, 54, 3215-3218. [CrossRef]

23. Bureau, S.; Ruizb, D.; Reich, M.; Gouble, B.; Bertrand, D.; Audergon, J.M. Application of ATR-FTIR for a rapid and simultaneous determination of sugars and organic acids in apricot fruit. Food Chem. 2009, 115, 1133-1140. [CrossRef]

24. Iñón, F.A.; Garrigues, J.M.; Garrigues, S.; Molina, A.; Guardiã, M. Selection of calibration set samples in determination of olive oil acidity by partial least squares-attenuated total reflectance-Fourier transform infrared spectroscopy. Anal. Chim. Acta 2003, 489, 59-75. [CrossRef]

25. Mata, P.; Vidalb, A.D.; Sendra, J.M.B.; Medinab, A.R.; Rodrígueza, L.C.; Cañada, M.J.A. Olive oil assessment in edible oil blends by means of ATR-FTIR and chemometrics. Food Cont. 2012, 23, 449-455. [CrossRef]

26. Cozzolino, D.; Cynkar, W.U.; Shah, N.; Smith, P. Multivariate data analysis applied to spectroscopy: Potential application to juice and fruit quality. Food Res. Int. 2011, 44, 1888-1896. [CrossRef]

27. Sivakesava, S.; Irudayaraj, J. A rapid spectroscopic technique for deter- mining honey adulteration with corn syrup. J. Food Sci. 2001, 66, 787-792. [CrossRef]

28. Sivakesava, S.; Irudayaraj, J. Classification of simple and complex sugar adulterants in honey by mid-infrared spectroscopy. Int. J. Food Sci. Technol. 2002, 37, 351-360. [CrossRef]

29. Sivakesava, S.; Irudayaraj, J. Detection of inverted beet sugar adulteration of honey by FTIR spectroscopy. J. Sci. Food. Agric. 2001, 81, 683-690. [CrossRef]

30. Kelly, J.D.; Petisco, C.; Downey, G. Application of Fourier transform mid-infrared spectroscopy to the discrimination between Irish artisanal Honey and such honey adulterated with various sugar syrups. J. Agric. Food Chem. 2006, 54, 6166-6171. [CrossRef]

31. Kelly, J.D.; Petisco, C.; Downey, G. Potential of near infrared transflectance spectroscopy to detect adulteration of Irish honey by beet invert syrup and high fructose corn syrup. JNIRS 2006, 14, 139-146. [CrossRef] 
32. Gallardo-Velazquez, T.; Osorio-Revilla, G.; Loa, M.Z.; Rivera- Espinoza, Y. Application of FTIR-HATR spectroscopy and multivariate analysis to the quantification of adulterants in Mexican honeys. Food Res. Int. 2009, 42, 313-318. [CrossRef]

33. Rios-Corripio, M.A.; Rios-Leal, E.; Rojas-López, M.; Delgado-Macuil, R. FTIR characterization of Mexican honey and its adulteration with sugar syrups by using chemometric methods. J. Phys. Conf. Ser. 2011, 274, 012098. [CrossRef]

34. Kelly, J.D.; Downey, G.; Fouratier, V. Initial study of honey adulteration by sugar solutions using midinfrared (MIR) spectroscopy and chemometrics. J. Agric. Food Chem. 2004, 52, 33-39. [CrossRef]

35. Anjos, O.; Iglesias, C.; Peres, F.; Martínez, J.; García, Á.; Taboada, J. Neural Networks Applied to Discriminate Botanical Origin of Honeys. Food Chem. 2015, 175, 128-136. [CrossRef] [PubMed]

36. Tewari, J.; Irudayaraj, J. Quantification of saccharides in multiple floral honeys using transform infrared micro attenuated total reflectance spectroscopy. J. Agric. Food Chem. 2004, 52, 3237-3243. [CrossRef] [PubMed]

37. Chung, H.; Ku, M.S.; Lee, J.S. Comparison of near-infrared and mid-infrared spectroscopy for the determination of distillation property of kerosene. Vib. Spec. 1999, 20, 155-163. [CrossRef]

38. Lichtenberg-Kraag, B.; Hedtke, C.; Bienefeld, K. Infrared spectroscopy in routine quality analysis of honey. Apidologie 2002, 33, 327-337. [CrossRef]

39. Ruoff, K.; Luginbühl, W.; Künzli, R.; Iglesias, M.T.; Bogdanov, S.; Bosset, J.O.; Von Der Ohe, K.; Von Der Ohe, W.; Amadò, R. Authentication of the Botanical and Geographical Origin of Honey by Front-Face Fluorescence Spectroscopy. J. Agric. Food Chem. 2006, 54, 6873-6880. [CrossRef]

40. Hennessy, S.; Downey, G.; O’Donnell, C. Multivariate Analysis of Attenuated Total Reflection-Fourier Transform Infrared Spectroscopic Data to Confirm the Origin of Honeys. Appl. Spectrosc. 2008, 62, 1115-1123. [CrossRef]

41. Woodcock, T.; Downey, G.; O'Donnell, C.P. Near Infrared Spectral Fingerprinting for Confirmation of Claimed PDO Provenance of Honey. Food Chem. 2009, 114, 742-746. [CrossRef]

42. De La Fuente, E.; Ruiz-Matute, A.I.; Valencia-Barrera, R.M.; Sanz, J.; Martínez Castro, I. Carbohydrate Composition of Spanish Unifloral Honeys. Food Chem. 2011, 129, 1483-1489. [CrossRef]

43. Eriksson, L.; Johansson, E.; Kettaneh-Wold, N.; Trygg, J.; Wikström, C.; Wold, S. Multi- and Megavariate Data Analysis (Part 2). Second ed. Vol. Advanced Applications and Method Extensions; Umetrics: Umea Sweden, 2006.

44. Farrés, M.; Platikanov, S.; Tsakovski, S.; Tauler, R. Comparison of the variable importance in projection (VIP) and of the selectivity ratio (SR) methods for variable selection and interpretation. J. Chemom. 2015, 29, 528-536. [CrossRef]

45. Pedregosa, F.; Varoquaux, G.; Gramfort, A.; Michel, V.; Thirion, B.; Grisel, O.; Blondel, M.; Prettenhofer, P.; Weiss, R.; Dubourg, V.; et al. Scikit-learn: Machine Learning in Python. JMLR 2011, 12, 2825-2830.

46. Movasaghi, Z.; Rehman, S.; Rehman, I. Fourier transform infrared (FTIR) spectroscopy of biological tissues. Appl. Spectrosc. Rev. 2008, 43, 134-179. [CrossRef]

47. Cai, S.; Singh, R.B. A distinct utility of the amide III infrared band for secondary structure estimation of aqueous protein solutions using partial least squares methods. Biochemistry 2004, 43, 2541-2549. [CrossRef] [PubMed]

48. Philip, D. Honey mediated green synthesis of gold nanoparticles. Spectrochim. Acta Part A Mol. Biomol. Spectrosc. 2009, 73, 650-653. [CrossRef] [PubMed]

(C) 2020 by the authors. Licensee MDPI, Basel, Switzerland. This article is an open access article distributed under the terms and conditions of the Creative Commons Attribution (CC BY) license (http://creativecommons.org/licenses/by/4.0/). 